\title{
Polyacrylonitrile nanofibers coated with silver nanoparticles using a modified coaxial electrospinning process
}

This article was published in the following Dove Press journal:

International Journal of Nanomedicine

9 November 2012

Number of times this article has been viewed

\author{
Deng-Guang Yu' \\ Jie Zhou ${ }^{2}$ \\ Nicholas P Chatterton ${ }^{3}$ \\ Ying $\mathrm{Li}^{\prime}$ \\ Jing Huang ${ }^{2}$ \\ Xia Wang \\ 'School of Materials Science and \\ Engineering, University of Shanghai \\ for Science and Technology, Shanghai, \\ People's Republic of China; ${ }^{2}$ School \\ of Life Sciences, East China Normal \\ University, Shanghai, People's \\ Republic of China; ${ }^{3}$ Faculty of Life \\ Sciences, London Metropolitan \\ University, London, United Kingdom
}

Correspondence: Jing Huang 3663 North Zhongshan Road, Science Building B8I3, Shanghai, 200062,

People's Republic of China

Tel +86 2l 62233295

Fax +86 21 62233295

Email jhuang@bio.ecnu.edu.cn

Xia Wang

516 Jungong Road, Yangpu District, Shanghai 200093, People's Republic of China

Tel +86 2I 55274069

Fax +86 2I 55270632

Email wangxia@usst.edu.cn
Background: The objective of this investigation was to develop a new class of antibacterial material in the form of nanofibers coated with silver nanoparticles (AgNPs) using a modified coaxial electrospinning approach. Through manipulation of the distribution on the surface of nanofibers, the antibacterial effect of Ag can be improved substantially.

Methods: Using polyacrylonitrile (PAN) as the filament-forming polymer matrix, an electrospinnable PAN solution was prepared as the core fluid. A silver nitrate $\left(\mathrm{AgNO}_{3}\right)$ solution was exploited as sheath fluid to carry out the modified coaxial electrospinning process under varied sheath-to-core flow rate ratios.

Results: Scanning electron microscopy and transmission electron microscopy demonstrated that the sheath $\mathrm{AgNO}_{3}$ solution can take a role in reducing the nanofibers' diameters significantly, a sheath-to-core flow rate ratio of 0.1 and 0.2 resulting in PAN nanofibers with diameters of $380 \pm 110 \mathrm{~nm}$ and $230 \pm 70 \mathrm{~nm}$ respectively. AgNPs are well distributed on the surface of PAN nanofibers. The antibacterial experiments demonstrated that these nanofibers show strong antimicrobial activities against Bacillus subtilis Wb800, and Escherichia coli $d h 5 \alpha$.

Conclusion: Coaxial electrospinning with $\mathrm{AgNO}_{3}$ solution as sheath fluid not only facilitates the electrospinning process, providing nanofibers with reduced diameters, but also allows functionalization of the nanofibers through coating with functional ingredients, effectively ensuring that the active antibacterial component is on the surface of the material, which leads to enhanced activity. We report an example of the systematic design, preparation, and application of a novel type of antibacterial material coated with AgNPs via a modified coaxial electrospinning methodology.

Keywords: antibacteria, nanofibers, nano-coating, coaxial electrospinning, Ag nanoparticles

\section{Introduction}

One of the aims of nanotechnology is the control of structures at the nanometer level. Such nanoengineering can lead to significantly modified, and often enhanced, properties. ${ }^{1}$ Electrospinning is a simple and straightforward technology for generating nanofibers. The popularity of this system is not only due to its easy implementation, versatility with regard to potential ingredient materials, but also its convenience in obtaining nanostructures. ${ }^{2-6}$

As a simple and straightforward "top-down" process, electrospinning has the unique capability of copying structures from the macro world to products at the micro/ nanoscale directly. ${ }^{7-10}$ For example, electrospinning can easily duplicate the structure of macro jet devices (as concentric and side-by-side spinnerets) to generate products with special nanostructures such as core-sheath and side-by-side nanofibers. ${ }^{7-10}$ 
In particular coaxial electrospinning, in which a concentric spinneret can accommodate two different liquids, is regarded as one of the most significant breakthroughs in the field. ${ }^{11-13}$ It has been applied in controlling secondary structures of nanofibers, encapsulating drugs or biological agents into the polymeric nanofibers, fabricating polymeric microtubes, preparing nanofibers from materials that lack filament-forming properties, and enclosing functional liquids within the fiber matrix. ${ }^{14-18}$ In traditional coaxial electrospinning, the sheath solution acts as a guide and surrounds the core material, which means that the sheath fluids must be electrospinnable and have enough viscosity to overcome the interfacial tension between the two solutions through "viscous dragging" and "contact friction" for a successful coaxial process. ${ }^{17,18}$

More recently, a modified process based on coaxial electrospinning was reported, in which only unspinnable solvents were used as sheath fluids. This process was exploited to manipulate nanofiber diameters and control the size of self-assembled nanoparticles generated from the fibers, to prepare fibers from concentrated polymer solutions previously thought to be unspinnable, and to further decrease the nanofiber diameter to take advantage of the resultant high surface area and porosity in the nonwoven mats. ${ }^{19-22}$ In all these investigations, sheath solvents act as a useful tool, permitting core electrospun fluid jets to be subjected to longer periods of electrical drawing. Later advances involved adding surfactants and salts to the sheath fluids so as to manipulate the conductivity and surface tension of sheath solutions for a better coaxial electrospinning process and produce nanofibers with even smaller diameters and a smooth surface morphology. ${ }^{23-29}$ However in all the abovementioned reports, the modified coaxial electrospinning processes generated nanofibers with improved quality in terms of nanofiber diameter, diameter distribution, as well as nanofiber morphology, but no attempts were made to modify the nanofibers so as to improve their functionality and enhance their nascent efficiency.

Silver (Ag) ions, colloidal silver nanoparticles (AgNPs) and silver-containing compounds are well documented as effective antiseptics for controlling broad-spectrum microbes and antibiotic-resistant bacteria in vitro. ${ }^{30-42}$ Electrospun Agcontaining polymer nanofiber mats have been intensively investigated for potential biomedical applications, such as wound-dressing materials, body wall repairs, augmentation devices, tissue scaffolds, and antimicrobial filters. ${ }^{43-47}$ However, all the nanofibers were prepared using a single fluid electrospinning approach involving codissolving solutions of polymer and silver nitrate $\left(\mathrm{AgNO}_{3}\right)$. This was then followed by reduction of the incorporated $\mathrm{Ag}^{+}$to $\mathrm{AgNPs}$ so as to take advantage of the unique property of nanofibers such as huge surface, small diameter, high porosity, and a three-dimensional continuous web structure. Although the prepared Ag-containing polymer nanofibers were demonstrated to provide the desired performance, most of the Ag was wasted as it was buried in the inner part of the nanofibers. It was proposed that if the AgNPs can be distributed on the surface of the nanofibers, the efficacy of the formulated material should be improved substantially. ${ }^{48-50}$

Polyacrylonitrile (PAN) is a synthetic, semicrystalline organic polymer resin, with the linear formula $\left(\mathrm{C}_{3} \mathrm{H}_{3} \mathrm{~N}\right)_{\mathrm{n}}$. It is a versatile polymer used to produce a large variety of products including ultrafiltration membranes, hollow fibers for reverse osmosis, fibers for textiles, oxidized flame retardant fibers, and carbon fiber. ${ }^{51}$ Based on its wide-ranging applications and good electrospinnability, PAN was selected as the filament-forming matrix. The PAN nanofibers with AgNPs distributed on their surface were produced using a modified coaxial electrospinning process, which involved using only $\mathrm{AgNO}_{3}$ solution as the sheath fluid to facilitate the electrospinning process and render the new functions of the nanofibers.

\section{Materials and methods \\ Materials}

PAN powders $\left(\bar{M}_{w}=80,000\right)$ were purchased from Jinshan Petrochemistry Co, Ltd (Shanghai, China). $\mathrm{N}, \mathrm{N}$-dimethylacetamide (DMAc) and $\mathrm{AgNO}_{3}$ were obtained from the Sinopharm Chemical Reagent Co, Ltd (Shanghai, China). All reagents were analytical grade and used without further purification. Water was double distilled just before use.

\section{Coaxial electrospinning process}

\section{Preparation of spinning solutions}

A solution of $15 \%(\mathrm{w} / \mathrm{v})$ PAN in DMAc was used as core fluid. Pure DMAc and a concentration of $10 \%(\mathrm{w} / \mathrm{v}) \mathrm{AgNO}_{3}$ in DMAc were employed as sheath fluids. Other conditions for fabrication of different nanofibers are listed in Table 1.

Table I Experimental parameters for the fabrication nanofibers

\begin{tabular}{lllll}
\hline Number & Process & Sheath fluid & \multicolumn{2}{c}{ Flow rate $(\mathbf{m L} / \mathbf{h})$} \\
\cline { 4 - 5 } & & & Sheath & Core \\
\hline FI & Single & In atmosphere & - & 1.0 \\
F2 & Coaxial & Pure DMAc & 0.2 & 1.0 \\
F3 & Coaxial & $\mathrm{AgNO}_{3}$ solution & 0.1 & 1.0 \\
F4 & Coaxial & $\mathrm{AgNO}_{3}$ solution & 0.2 & 1.0 \\
\hline
\end{tabular}

Abbreviations: $\mathrm{AgNO}_{3}$, silver nitrate; DMAc, $\mathrm{N}, \mathrm{N}$-dimethylacetamide. 
The concentric spinneret was designed and fabricated in-house and used to carry out coaxial electrospinning. Two syringe pumps (KDS100 and KDS200; Cole-Parmer ${ }^{\circledR}$, Chicago, IL) were employed to drive the sheath and core fluids according to the parameters in Table 1. A metal clip was used to connect the spinneret with the high voltage supply (Shanghai SuTe Electrical Co, Ltd, Shanghai, China) and the applied voltage was $18 \mathrm{kV}$. The nanofibers were collected on a metal collector wrapped with aluminum foil and kept at a fixed distance of $20 \mathrm{~cm}$ away from the needle tip of the spinneret. The coaxial processes were recorded using a digital video recorder (PowerShot A640; Canon, Tokyo, Japan) under magnifications of $11 \times$. The electrospun nanofibers were exposed to $254 \mathrm{~nm}$ ultraviolet light for 24 hours to reduce the $\mathrm{Ag}^{+}$to AgNPs.

\section{Characterization}

The morphology of the core-sheath nanofibers was assessed using a Quanta FEG450 scanning electron microscope (SEM; FEI Corporation, Eindhoven, The Netherlands). The average fiber diameter was determined by measuring diameters of nanofibers over 100 points from field emission SEM images using Image J software (National Institutes of Health, Bethesda, MD). The elemental composition of the samples was analyzed by an energy-dispersive spectrometer EDAX (EDS; EDAX Inc, Draper, UT), [software; IE300X, Oxford, UK] attached to the SEM.

Transmission electron microscopy (TEM) images of the samples were recorded on a JEM 2100F field emission TEM (JEOL, Tokyo, Japan). TEM samples were collected by fixing a lacy carbon-coated copper grid on the collector.

\section{Antibacterial test}

The in vitro antibacterial activities of PAN nanofibers F2, F3, and F4 were examined according to enumeration by the plate count method described previously. ${ }^{52,53}$ The following microorganisms were used: Gram-positive Bacillus subtilis ( $\mathrm{Wb800)}$ and Gram-negative Escherichia coli dh $5 \alpha$ (E. coli $d h 5 \alpha$ ). Briefly, sterilized Luria-Bertani (LB) broth was measured $(5 \mathrm{~mL})$ into an Erlenmeyer flask. Sixty $\mathrm{mg}$ PAN nanofibers were introduced into the LB broth solution, which contained approximately $1.5 \times 10^{5}$ colony-forming units (CFU) of $W b 800$ and $E$. coli $d h 5 \alpha$, respectively. The mixtures were cultured at $37^{\circ} \mathrm{C}$ in a shaking incubator for 12 hours. One hundred microliters of each of these cell solutions was seeded onto LB agar using a surface spread plate technique. The plates were incubated at $37^{\circ} \mathrm{C}$ for 24 hours. The numbers of bacterial colonies (CFU) were counted.
Pure phosphate-buffered saline (PBS) and $\mathrm{AgNO}_{3}$ were also tested as blank control and positive control, respectively. One hundred microliters of each of these cell solutions was seeded onto LB agar using a surface spread plate technique. The plates were incubated at $37^{\circ} \mathrm{C}$ for 24 hours. The numbers of bacterial colonies (CFU) were counted. The counts were used to calculate the surviving number of bacteria. The antibacterial efficacy (ABE in \%) of the specimen was calculated according to the following equation:

$$
\operatorname{ABE}(\%)=\left(V_{c}-V_{t}\right) / V_{c} \times 100
$$

where $V_{c}$ and $V_{t}$ stands for the numbers of viable bacterial colonies of the blank control (pure PBS buffer added) and test specimen, respectively. All the experiments were repeated six times and the results are presented as mean values.

\section{Results and discussion Modified coaxial electrospinning}

A schematic diagram of the coaxial electrospinning process is shown in Figure 1A. Two syringe pumps were employed to drive the sheath and core fluids respectively. The nanofibers are collected on a plate and the coaxial processes can be recorded using a digital video recorder. The self-made concentric spinneret ${ }^{20}$ used to carry out the modified coaxial electrospinning is shown in Figure 1B. The upper surface of the core capillary was indented $1.0 \mathrm{~mm}$ from the tip of the sheath capillary, and this facilitates easier envelopment of the core spinning solution by the outer solvent. The system differs from conventional coaxial spinnerets in which the tips of the inner and the outer capillary are coplanar or the tips of the inner capillary slightly project out the tips of the outer capillary.

The critical voltage applied to a fluid to initiate Taylor cone formation and the straight thinning jet $\left(V_{c}\right)$ has a close
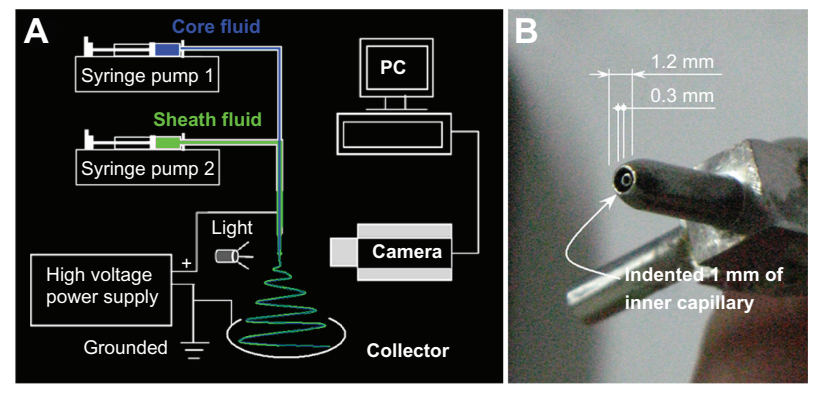

Figure I Schematic diagram of the modified coaxial electrospinning process (A) and the homemade concentric spinneret (B). 
relationship with the diameter of the sheath part of the concentric spinneret: ${ }^{54}$

$$
V_{c} \sim \sqrt{\frac{\gamma d^{2}}{\varepsilon R}}
$$

where $V_{c}$ is the critical voltage for a jet emanating from the meniscus tip, $d$ is the electrode separation, $\varepsilon$ is the permittivity, $\gamma$ is the surface tension, and $R$ is the principal curvature of the liquid meniscus. A low diameter spinneret orifice gives a high value of $R$, and thus only a small $V_{c}$ is needed to initiate electrospinning. The self-made spinneret used in this work has outside and inner diameters of 1.2 and $0.3 \mathrm{~mm}$ respectively, facilitating the coaxial electrospinning process.

A digital picture of the coaxial electrospinning process is shown in Figure 2A. Figure 2B shows the connection of power supply with the spinneret and the sheath and core fluids. This modified coaxial electrospinning differs from the traditional coaxial process in that the core fluid was the protagonist whereas the sheath fluid played a supporting role for smoothing and stabilizing the overall process. It also differs from the traditional single fluid process as the formation of the Taylor cone, the thinning of straight jet and part of the instability region would occur in the surrounding solvent, not the atmosphere.

Figure $2 \mathrm{C}$ exhibits a typical fluid jet trajectory when core and sheath flow rates of 1.0 and $0.2 \mathrm{~mL} /$ hour were taken under a high voltage of $15 \mathrm{kV}$. A typical electrospinning process can be discerned according to the following stages: (1) fluid charging and the formation of the compound Taylor cone-jet, (2) thinning of the straight fluid jet, (3) onset and growth of jet instabilities (ie, the bending and whipping region) and random collections of the nanofibers. When the applied voltages were further raised to $18 \mathrm{kV}$, the compound Taylor cone shrank into the indented region of the outer capillary of spinneret and cannot be discerned in the picture (Figure 2D).
However, the straight fluid jets, the bending and whipping of the fluid jets under the electrical field are all similar as those in Figure 2C. A higher applied voltage always leads to finer nanofibers, thus a voltage of $18 \mathrm{kV}$ was selected as the final value for producing Ag-coating PAN nanofibers.

\section{Morphology}

Figure 3 shows the SEM images of the prepared four types of nanofibers. All the nanofibers have uniform structures without beads-on-a-string morphology owing to the good electrospinnability of the core PAN solutions. However, PAN nanofibers from the traditional single fluid electrospinning F1 have very rough surfaces, along with the largest average diameters and broadest distributions (750 $\pm 160 \mathrm{~nm}$; Figure 3A).

When the coaxial electrospinning was taken with DMAc as the sheath fluid, the traditional Taylor cone-air and fluid jet-air interfaces have become Taylor cone-solvent and fluid jet-solvent interfaces. As a result, the formation of the cone-jet, the thinning of the straight jet and partial bending and whipping were different when compared to the traditional system conducted in air. The presence of sheath DMAc in the process would not only let the PAN fluid jet be subjected to a longer period of electrical drawing, but also provide stable and robust conditions for the solvent in the PAN fluid jet to evaporate. Correspondingly, the modified process generated nanofibers F2 with smaller diameters, narrower distributions (480 $\pm 130 \mathrm{~nm})$, and smooth surface morphology (Figure 3B).

When $\mathrm{AgNO}_{3}$ solution was employed as sheath fluid, the nanofiber (F3) diameters and their distributions (Figure 3C) even became smaller and narrower $(380 \pm 130 \mathrm{~nm})$ although a smaller sheath-to-core fluid rate ratio was taken. This could be attributed to the increase of conductivity of the sheath solution that contained the electrolyte $\mathrm{AgNO}_{3}$. As anticipated,
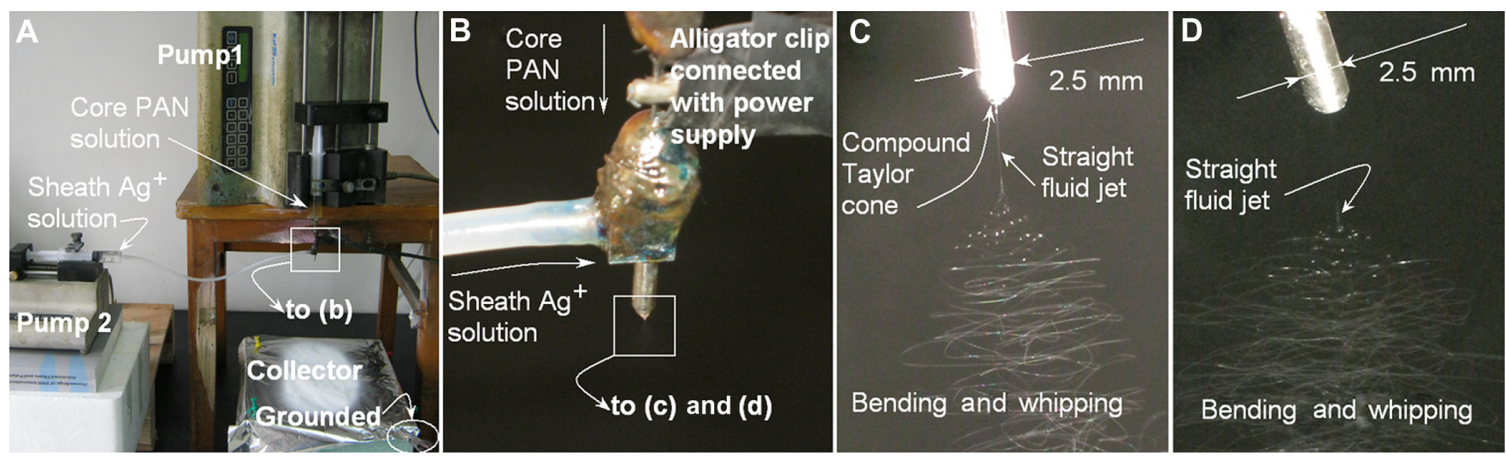

Figure 2 Digital pictures of the modified coaxial process: (A) A digital picture shows arrangement of apparatus; (B) The connection of power supply with the spinneret and the sheath and core fluids; (C and D) A typical fluid jet trajectory (taken with a magnification of $12 \times$, a core and sheath flow rates of 1.0 and $0.2 \mathrm{~mL} /$ hour and a fiber collection distance of $20 \mathrm{~cm}$ ) under a high voltage of 15 and $18 \mathrm{kV}$, respectively.

Abbreviation: PAN, Polyacrylonitrile. 


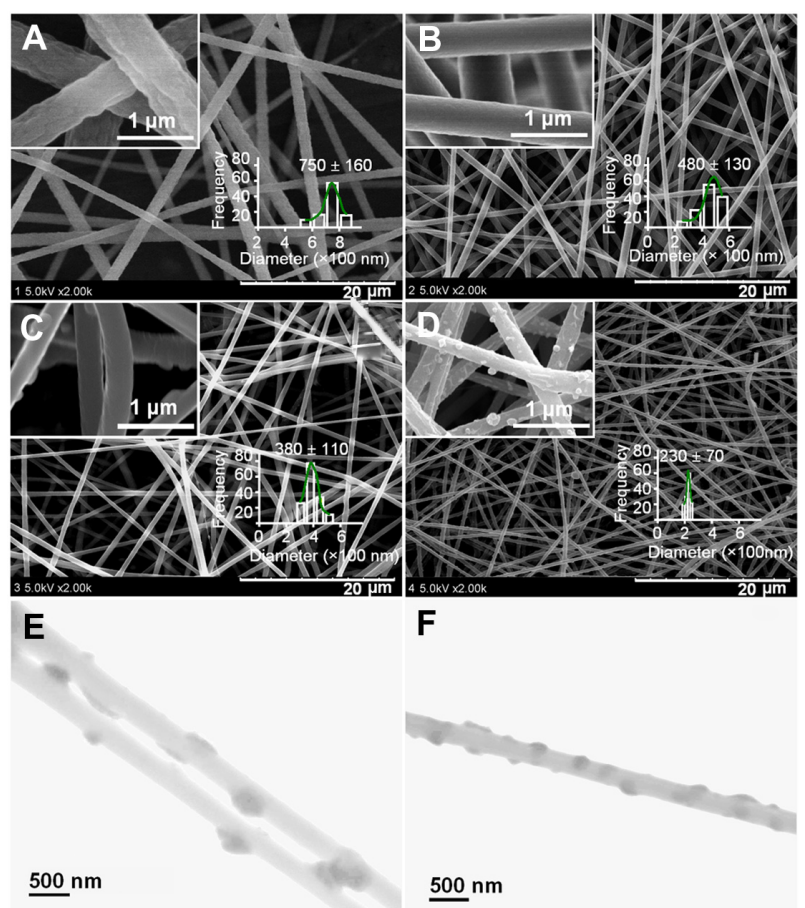

Figure 3 SEM images of PAN nanofibers prepared using different processes: (A) surface morphologies and diameter distribution of nanofibers $\mathrm{FI}$ from single fluid electrospinning; (B) surface morphologies and diameter distribution of nanofibers F2 from a modified coaxial process with only DMAc as sheath fluid; ( $\mathbf{C}$ and $\mathbf{D}$ ) surface morphologies and diameter distribution of nanofibers F3 and F4 from a modified coaxial process with $\mathrm{AgNO}_{3}$ solution as sheath fluid, respectively. The scale bars in the insets of (C and $\mathbf{D})$ represent $500 \mathrm{~nm}$. (E and $\mathbf{F}$ ) are TEM images of nanofibers $\mathrm{F} 3$ and $F 4$, respectively.

Abbreviations: $\mathrm{AgNO}_{3}$, silver nitrate; DMAc, $\mathrm{N}, \mathrm{N}$-dimethylacetamide; PAN, polyacrylonitrile; SEM, scanning electron microscopy; TEM, transmission electron microscopy.

the increase of sheath-to-core fluid rate ratio from 0.1 to 0.2 resulted in the nanofibers F4 (Figure 3D) with the smallest diameter and narrowest distribution $(230 \pm 70 \mathrm{~nm})$.

As shown in the insets of Figure $3 \mathrm{C}$ and $\mathrm{D}$, the reduced $\mathrm{AgNPs}$ were distributed on the surface of nanofibers F3 and F4. It is surprising that the AgNPs on the surface of nanofibers $\mathrm{F} 3$ have a diameter range of 50 to $100 \mathrm{~nm}$, larger than those on the surface of nanofibers F4, which have a diameter range of 20 to $80 \mathrm{~nm}$. When a sheath-to-core fluid rate ratio of 0.1 was used to conduct the modified coaxial electrospinning, the smaller flow of $\mathrm{AgNO}_{3}$ solution was not able to follow the core PAN fluid jet during the fast bending and whipping process. This results in a separation of the sheath $\mathrm{AgNO}_{3}$ solution and a nonuniform deposition of $\mathrm{AgNO}_{3}$ on the surface of nanofibers. Thus, a higher sheathto-core fluid rate ratio of 0.2 should result in a more homogeneous distribution of $\mathrm{AgNO}_{3}$ on the surface of nanofibers $\mathrm{F} 4$ compared to that on the surface of nanofibers F3. When the $\mathrm{Ag}^{+}$on the surface of PAN nanofibers was reduced to AgNPs, those on the surface of nanofibers F4 had a more uniform size and a tighter distribution than those on the surface of nanofibers F3. It is anticipated that reduction of $\mathrm{Ag}^{+}$to AgNP on the surface of nanofibers should be beneficial to long-term stability of the system and prevent Ag leaching from the fibers.

Throughout the modified coaxial electrospinning, the sheath $\mathrm{Ag}^{+}$solution would exert the following influences on the process (Figure 4A): (1) facilitating the formation of the Taylor cone due to lower surface tensions and high electrical conductivity; (2) surrounding the straight thinning jet of the core PAN solutions to prevent fast evaporation of the core solvent, while the sheath solvent itself outwardly evaporates to the open air; (3) following the core fluid to enter the bending and whipping region. The primary reason that the sheath solution acts to thin the nanofibers is that it prevents evaporation of solvents from the surface of the core spinning polymer solutions prematurely, and in turn retains the core jet in a fluid state thus allowing it to be subjected to electrical drawing for a longer period in the unstable region. ${ }^{26-28}$

During the modified process, the electrospinnable core fluid jets had sufficient viscoelastic forces to balance Coulomb forces so allowing an even and continuous drawing. However, the sheath $\mathrm{Ag}^{+}$solution should break up into separate segments along the core PAN fluid jets due to lack of viscoelasticity at a certain place in the bending and whipping region as determined by the sheath rate ratio. When a relatively small sheath flow rate was taken, such as the case of nanofibers F3 in Figure 4B, the separation of sheath solutions on the surface of core fluid would be a little easier when the fluid jet still has a bigger size, which in turn formed larger and fewer AgNPs when they were reduced using ultraviolet light. When a relatively big sheath flow rate was used, such as the case of nanofibers F4 in Figure 4C, the surrounding Ag+

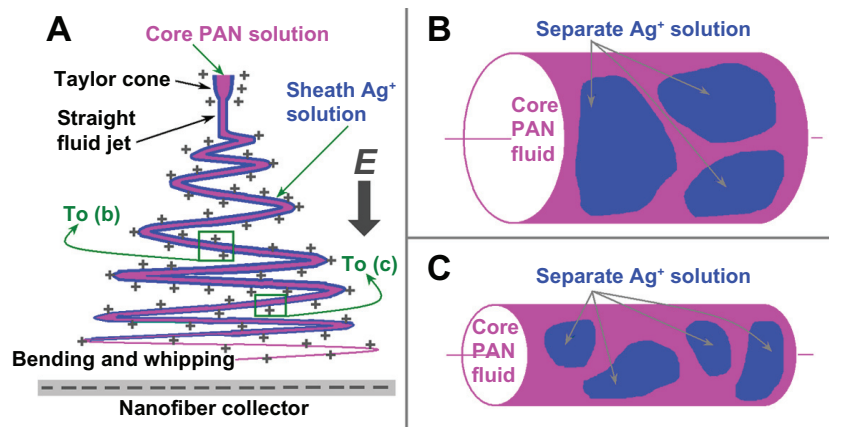

Figure $4 \mathrm{~A}$ schematic diagram of the influence of the sheath flow rate on the formation of silver nanoparticles (AgNPs): (A) a typical fluid jet trajectory during electrospinning; (B) an illustration of AgNPs on the surface of nanofibers $\mathrm{F} 3$ when a smaller sheath-to-core flow rate ratio was exploited; (C) an illustration of AgNPs on the surface of nanofibers F4 when a relatively larger sheath-to-core flow rate ratio was exploited.

Abbreviation: PAN, Polyacrylonitrile. 


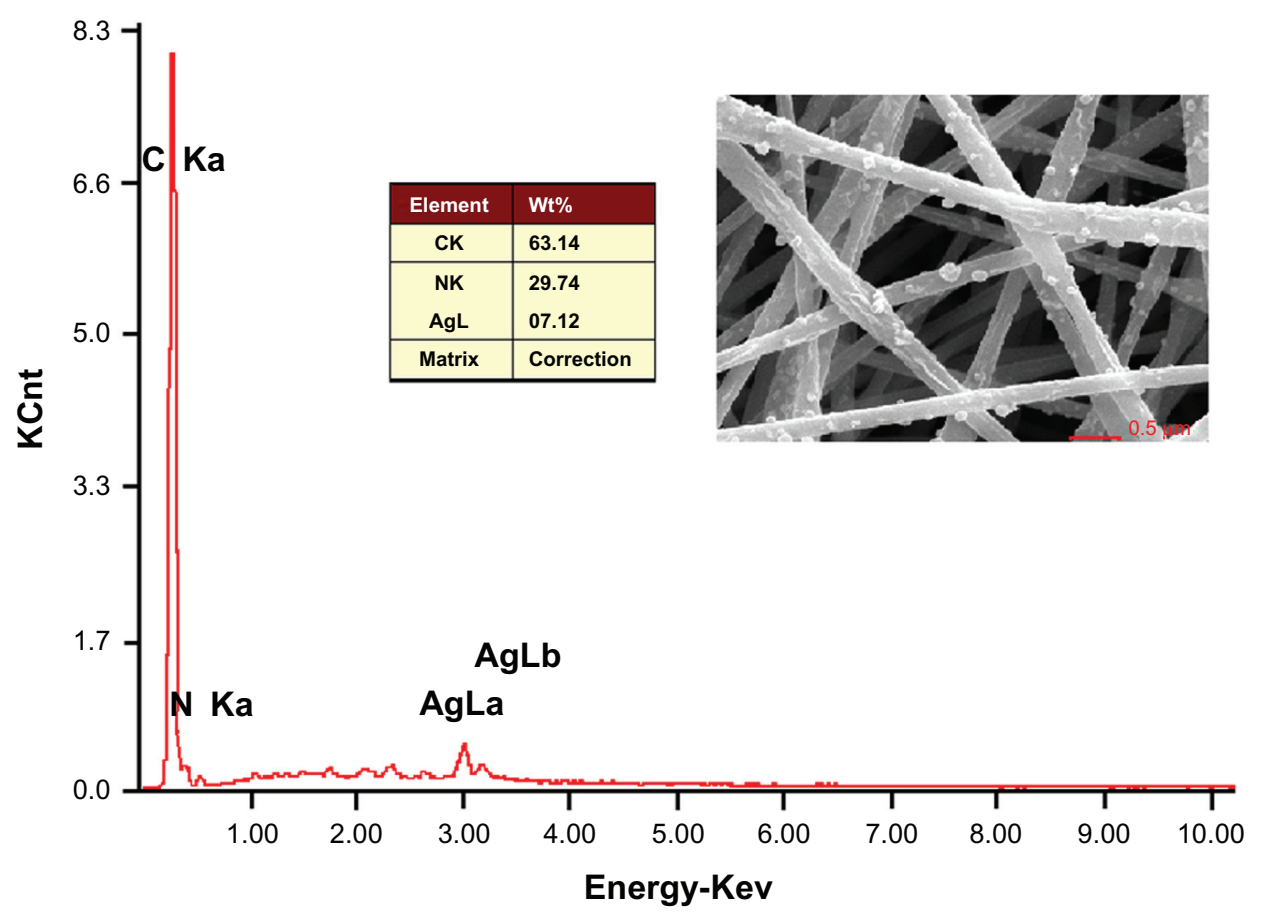

Figure 5 EDS spectrum of the Ag-containing electrospun PAN nanofibers F4. Abbreviations: EDS, energy-dispersive spectrometer; PAN, polyacrylonitrile.

solutions remained with the core PAN fluid for a longer time to enter the late period of the bending and whipping when the fluid jets had a smaller diameter. Thus correspondingly the sheath solutions were divided into smaller segments on the core PAN fluid jets, which in turn formed smaller and more AgNPs when they were reduced.

The EDS of the nanofibers F4 indicate the presence of Ag on the surface of the fibers (Figure 5). The content of AgNPs in the PAN nanofibers was $7.12 \%$ by weight. The theoretical content of $\mathrm{Ag}$ in the nanofibers can be calculated from the electrospinning conditions: ie, $(0.2 \times 10 \%) \times(108 / 170) /$ $[(0.2 \times 10 \%) \times(108 / 170)+1 \times 15 \%]=7.81 \%$. The EDS result demonstrates that nearly all the AgNPs were successfully immobilized on the surface of PAN nanofibers F4.

\section{Antibacterial activity}

The antibacterial capacities of AgNPs-coated PAN nanofibers F3 and F4 against Gram-positive $W b 800$ and Gram-negative E. coli $d h 5 \alpha$ were explored by the viable cell counting method. Their abilities in inhibiting the growth of the test bacteria are shown in Table 2. The ABE of the nanofibers F4 against $W b 800$ and $E$. coli $d h 5 \alpha$ were over $99.9 \%$. The ABE of the nanofibers F3 against $W b 800$ were also over $99.9 \%$, and that against $E$. coli $d h 5 \alpha$ was about $99.8 \%$. Pure PAN nanofibers $\mathrm{F} 2$ did not show any antibacterial properties. The AgNPs distributed on the surface of PAN nanofibers F3 and
F4 were responsible for the strong antibacterial activity of the nanofibers.

Previous electrospun polymer nanofibers have been employed as AgNPs-loaded matrices for the development antimicrobial materials. However, most of the functional Ag was buried in the inner part of the nanofibers and often a high content of $\mathrm{Ag}$ (up to $30 \%$ ) was used in the formulation when single fluid electrospinning was exploited. ${ }^{46}$ In contrast, the present study showed excellent antibacterial efficacies with only a content of less than $4 \%(\mathrm{w} / \mathrm{w}) \mathrm{Ag}$ in the nanofibers

Table 2 The antibacterial effect of AgNPs-coated PAN nanofiber against Wb800 and Escherichia coli dh5 $\alpha(\mathrm{n}=6)$

\begin{tabular}{lllll}
\hline Bacteria & Materials & Initial CFUa & $\begin{array}{l}\text { CFU after } \\
\text { I2 hours }\end{array}$ & ABE $^{\mathrm{b}}$ \\
\hline Wb800 & $\mathrm{AgNO}_{3}{ }^{\mathrm{c}}$ & $1.5 \times 10^{5}$ & $\leq 10$ & $>99.9$ \\
& $\mathrm{~F} 2$ & $1.5 \times 10^{5}$ & $6.6 \times 10^{6}$ & 0 \\
& $\mathrm{~F} 3$ & $1.5 \times 10^{5}$ & $2.8 \times 10^{3}$ & $>99.9$ \\
& $\mathrm{~F} 4$ & $1.5 \times 10^{5}$ & $1.8 \times 10^{3}$ & $>99.9$ \\
& $\mathrm{Blank}$ & $1.5 \times 10^{5}$ & $6.4 \times 10^{6}$ & - \\
E. coli $\mathrm{DH} 5 \alpha$ & $\mathrm{AgNO}_{3}{ }^{\mathrm{c}}$ & $1.5 \times 10^{5}$ & $\leq 10$ & $>99.9$ \\
& $\mathrm{~F} 2$ & $1.5 \times 10^{5}$ & $7.1 \times 10^{6}$ & 0 \\
& $\mathrm{~F} 3$ & $1.5 \times 10^{5}$ & $1.2 \times 10^{4}$ & 99.8 \\
& $\mathrm{~F} 4$ & $1.5 \times 10^{5}$ & $7.5 \times 10^{2}$ & $>99.9$ \\
& Blank & $1.5 \times 10^{5}$ & $7.0 \times 10^{6}$ & - \\
\hline
\end{tabular}

Note: ${ }^{2}$ The colony forming units. ${ }^{\text {T} T h e ~ a n t i-b a c t e r i a l ~ e f f i c a c y ~(\%) . ~}{ }^{\text {CT }}$ The weight of $\mathrm{AgNO} 3$ is $8 \mathrm{mg}$, equivalent to the content of $\mathrm{AgNO} 3$ in $60 \mathrm{mg}$ nanofibers $\mathrm{F} 4$.

Abbreviations: $\mathrm{ABE}$, antibacterial efficacy; $\mathrm{AgNO}_{3}$, silver nitrate; $\mathrm{AGNPs}$, silver nanoparticles; CFU, colony-forming units; PAN, polyacrylonitrile. 
(nanofibers F3) generated using a modified coaxial process. The position of AgNPs on the PAN nanofiber surface could endow the PAN nanofibers with excellent antibacterial capacities, and avoid wasting the noble metal buried in the inner part of the nanofibers by traditional electrospinning processes.

\section{Conclusion}

A modified coaxial electrospinning process was developed successfully to prepare a new type of antibacterial nanofiber in which AgNPs were distributed only on the surface of nanofibers to improve their antibacterial capability.

The nanofibers were prepared using a modified coaxial process and resulted in nanofibers that exhibited better quality than those obtained from the single fluid electrospinning in terms of nanofiber diameter, diameter distribution, and surface morphology. Under a sheath-to-core flow rate ratio of 0.2 , the resultant PAN nanofibers have a diameter of $230 \pm 70 \mathrm{~nm}$ with the AgNPs uniformly distributed on their surface. The antibacterial experiments demonstrated that both nanofibers F3 and F4 exhibited strong antimicrobial activities against $B$. subtilis $W b 800$ and $E$. coli $d h 5 \alpha$ even with a low Ag content present. Our study provides an example of the systematic design, preparation, and application of a novel type of structural antibacterial materials coated with AgNPs via a modified coaxial electrospinning.

\section{Acknowledgments}

This work was supported by the Key project of Shanghai Municipal Education Commission (No 13ZZ113) and National Science Foundation of China (Nos 31170920 , 51173107, and 21101107).

\section{Disclosure}

The authors report no conflicts of interest in this work.

\section{References}

1. Webster TJ. Nanomedicine: what's in a definition? Int J Nanomedicine. 2006;1:115-116.

2. Seil JT, Webster TJ. Spray deposition of live cells throughout the electrospinning process produces nanofibrous three-dimensional tissue scaffolds. Int J Nanomedicine. 2011;6:1095-1099.

3. Jannesari M, Varshosaz J, Morshed M, Zamani M. Composite poly(vinyl alcohol)/poly(vinyl acetate) electrospun nanofibrous mats as a novel wound dressing matrix for controlled release of drugs. Int J Nanomedicine. 2011;6:993-1003.

4. Neves NM, Campos R, Pedro A, Cunha J, Macedo F, Reis RL. Patterning of polymer nanofiber meshes by electrospinning for biomedical applications. Int J Nanomedicine. 2007;2:433-448.

5. Wang Y, Zhang C, Zhang Q, Li P. Composite electrospun nanomembranes of fish scale collagen peptides/chito-oligosaccharides: antibacterial properties and potential for wound dressing. Int J Nanomedicine. 2011;6: $667-676$.
6. Teo WE, Ramakrishna S. A review on electrospinning design and nanofiber assemblies. Nanotechnology. 2006;17:89-106.

7. Zhao Y, Cao X, Jiang L. Bio-mimic multichannel microtubes by a facile method J Am Chem Soc. 2007;29:764-765.

8. Yu JH, Fridrikh SV, Rutledge GC. Production of submicrometer diameter fibers by two-fluid electrospinning. Adv Mater. 2004;16:1562-1566.

9. Liu Z, Sun DD, Guo P, Leckie JO. An efficient bicomponent $\mathrm{TiO}_{2} / \mathrm{SnO}_{2}$ nanofiber photocatalyst fabricated by electrospinning with a side-by-side dual spinneret method. Nano Lett. 2007;7:1081-1085.

10. Yarin AL, Zussman E, Wendorff JH, Greiner A. Material encapsulation and transport in core-shell micro/nanofibers, polymer and carbon nanotubes and micro/nanochannels. J Mater Chem. 2007;17: 2585-2599.

11. Dzenis Y. Spinning continuous fibers for nanotechnology. Science. 2004;304:1917-1919.

12. Locertales IG, Barrero A, Guerrero I, Cortijo R, Marquez M, Ganan-Calvo AM. Micro/nano encapsulation via electrified coaxial liquid jets. Science. 2002;295:1695-1698.

13. Xin Y, Huang Z, Li W, Jiang Z, Tong Y, Wang C. Core-sheath functional polymer nanofibers prepared by co-electrospinning. Eur Polym J. 2008;44:1040-1045.

14. Zhang JF, Yang DZ, Xu F, Zhang ZP, Yin RX, Nie J. Electrospun core-shell structure nanofibers from homogeneous solution of poly(ethyleneoxide)/chitosan. Macromolecules. 2009;42: 5278-5284.

15. Park CH, Lee J. One-step immobilization of protein-encapsulated core/shell particles onto nanofibers. Macromol Mater Eng. 2010;295:544-550.

16. Sill TJ, Von Recum HA. Electrospinning: applications in drug delivery and tissue engineering. Biomaterials. 2008;29:1989-2006.

17. Moghe K, Gupta BS. Co-axial electrospinning for nanofiber structures: preparation and applications. Polym Rev. 2008;48:353-377.

18. Dror Y, Salalha W, Avrahami R, et al. One-step production of polymeric microtubes by co-electrospinning. Small. 2007;3:1064-1073.

19. Yu DG, Branford-White C, Chatterton NP, et al. Electrospinning of concentrated polymer solutions. Macromolecules. 2010;43: 10743-10746.

20. Yu DG, Branford-White C, Bligh SWA, White K, Chatterton NP, Zhu LM. Improving polymer nanofiber quality using a modified coaxial electrospinning process. Macromol Rapid Commun. 2011;32: 744-750.

21. Yu DG, Zhu LM, Branford-White C, Bligh SWA, White K. Coaxial electrospinning with organic solvent for controlling the self-assembled nanoparticle size. Chem Commun. 2011;47:1216-1218.

22. Yu DG, Branford-White C, White $\mathrm{K}$, et al. A modified coaxial electrospinning for preparing fibers from a high concentration polymer solution. eXPRESS Polym Lett. 2011;5:732-741.

23. Yu DG, Lu P, Branford-White C, Yang JH, Wang X. Polyacrylonitrile nanofibers prepared using co-axial electrospinning with $\mathrm{LiCl}$ solution as sheath fluid. Nanotechnology. 2011;22:435301.

24. Yu DG, White K, Yang JH, Wang X, Qian W, Li Y. PVP nanofibers prepared using co-axial electrospinning with salt solution as sheath fluid. Mater Lett. 2012;67:78-80.

25. Yu DG, Yang JM, Li L, Lu P, Zhu LM. Obtaining finer polymer nanofibers using two different electrospinning processes. Fiber Polym. 2012; 13:450-455.

26. Yu DG, Williams GR, Gao LD, Annie Bligh SW, Yang JH, Wang X. Coaxial electrospinning with sodium dodecylbenzene sulfonate solution for high quality polyacrylonitrile nanofibers. Colloid Surf A. 2012;396: $161-168$.

27. Yang JM, Yu DG. Co-axial electrospinning with sodium thiocyanate solution for preparing polyacrylonitrile nanofibers. J Polym Res. 2012;19:9789

28. Yu DG, Chatterton NP, Yang JH, Wang X, Liao YZ. Coaxial electrospinning with Triton X-100 solutions as sheath fluids for preparing PAN nanofibers. Macromol Mater Eng. 2012;297:395-401.

29. Yu DG, Yu JH, Chen L, Williams GR, Wang X. Modified coaxial electrospinning for the preparation of high-quality ketoprofen-loaded cellulose acetate nanofibers. Carbohydr Polym. 2102;90:1016-1023. 
30. Eid KAM, Azzazy HME. Controlled synthesis and characterization of hollow flower-like silver nanostructures. Int J Nanomedicine. 2012;7:1543-1550.

31. Allahverdiyev AM, Abamor ES, Bagirova M, et al. Antileishmanial effect of silver nanoparticles and their enhanced antiparasitic activity under ultraviolet light. Int J Nanomedicine. 2011;6:2705-2714.

32. Chen M, Yang Z, Wu H, Pan X, Xie X, Wu C. Antimicrobial activity and the mechanism of silver nanoparticle thermosensitive gel. Int $J$ Nanomedicine. 2011;6:2873-2877.

33. Jang S, Park JW, Chal HR, et al. Silver nanoparticles modify VEGF signaling pathway and mucus hypersecretion in allergic airway inflammation. Int J Nanomedicine. 2012;7:1329-1343.

34. Singh SK, Goswami K, Sharma RD, Reddy MVR, Dash D. Novel microfilaricidal activity of nanosilver. Int J Nanomedicine. 2012; 7: 1023-1030.

35. Bei Y, Chen X, Liu Y, et al. Novel norcantharidin-loaded liver targeting chitosan nanoparticles to enhance intestinal absorption. Int $J$ Nanomedicine. 2012;7:1819-1827.

36. Su YH, Yin ZF, Xin HL, et al. Optimized antimicrobial and antiproliferative activities of titanate nanofibers containing silver. Int $J$ Nanomedicine. 2011;6:1579-1586.

37. Seil JT, Webster TJ. Antimicrobial applications of nanotechnology: methods and literature. Int J Nanomedicine. 2012;7:2767-2781.

38. Hu G, Xiao L, Tong P, et al. Antibacterial homeostatic dressings with nanoporous bioglass containing silver. Int J Nanomedicine. 2012;7:2613-2620.

39. Elzatahry AA, A1-Enizi AM, Elsayed EA, et al. Nanofiber composites containing N-heterocyclic carbine complexes with antimicrobial activity. Int J Nanomedicine. 2012;7:2829-2832.

40. Jena P, Mohanty S, Mallick R, Jacob B, Sonawane A. Toxicity and antibacterial assessment of chitosan coated silver nanoparticles on human pathogens and macrophage cells. Int J Nanomedicine. 2012;7: 1805-1818.

41. Chiao SH, Lin SH, Shen CI, et al. Efficacy and safety of nanohybrids comprising silver nanoparticles and silicate clay for controlling Salmonella infection. Int J Nanomedicine. 2012;7:2421-2432.

42. Sharma VK, Yngard RA, Lin Y. Silver nanoparticles: Green synthesis and their antimicrobial activities. Adv Colloid Interfac Sci. 2009;145:83-96.
43. Lim SK, Lee SK, Hwang SH, Kim H. Photocatalytic deposition of silver nanoparticles organic/inorganic composite nanofibers. Macromol Mater Eng. 2006;291:1265-1270.

44. Son WK, Youk JH, Lee TS, Park WH. Preparation of Antimicrobial ultrafine cellulose acetate fibers with silver nanoparticles. Macromol Rapid Commun. 2004;25:1632-1637.

45. Chou WL, Yu DG, Yang MC. The preparation and characterization of silver-loading cellulose acetate hollow fiber membrane for water treatment. Polym Adv Technol. 2005;16:600-607.

46. Xu X, Yang Q, Wang Y, Yu H, Chen X, Jing X. Biodegradable electrospun poly(L-lactide) fibers containing antibacterial silver nanoparticles. Euro Polym J. 2006;42:2081-2087.

47. Hong KH. Preparation and properties of electrospun poly (vinyl alcohol)/silver fiber web as wound dressings. Polym Eng Sci. 2007;47: 43-49.

48. Carlberg B, Ye LL, Liu J. Surface-confined synthesis of silver nanoparticle composite coating on electrospun polyimide nanofibers. Small. 2011;7:3057-3066.

49. Yu LN, Zhao J, Shen LD, Gao Y, Wang XF. Ag-coated PAN nanofibers prepared by poly(dopamine)-assisted electroless plating. Adv Mater Res. 2012;482-484:2543-2546.

50. Zhao P, Fan J. Silver polyhedron coated electrospun nylon 6 nano-fibrous membrane with good infrared extinction, ultraviolet shielding and water vapor permeability. J Appl Polym Sci. 2012;124:5138-5144.

51. Wikipedia. Polyacrylonitrile. Available from: http://en.wikipedia.org/ wiki/Polyacrylonitrile. Accessed on September 10, 2012.

52. Melaiye A, Sun Z, Hindi K, et al. Silver(I)-imidazole cyclophane gemdiol complexes encapsulated by electrospun tecophilic nanofibers: Formation of nanosilver particles and antimicrobial activity. J Am Chem Soc. 2005;127:2285-2291.

53. Son WK, Youk JH, Lee TS, Park WH. Preparation of antimicrobial ultrafine cellulose acetate fibers with silver nanoparticles. Macromol Rapid Commun. 2004;25:1632-1637.

54. Yeo LY, Friend JR. Electrospinning carbon nanotube polymer composite nanofibers. J Exp Nanosci. 2006;2:177-209.
International Journal of Nanomedicine

\section{Publish your work in this journal}

The International Journal of Nanomedicine is an international, peerreviewed journal focusing on the application of nanotechnology in diagnostics, therapeutics, and drug delivery systems throughout the biomedical field. This journal is indexed on PubMed Central, MedLine, CAS, SciSearch $\AA$, Current Contents ${ }^{\circledR} /$ Clinical Medicine,

\section{Dovepress}

Journal Citation Reports/Science Edition, EMBase, Scopus and the Elsevier Bibliographic databases. The manuscript management system is completely online and includes a very quick and fair peer-review system, which is all easy to use. Visit http://www.dovepress.com/ testimonials.php to read real quotes from published authors. 